Article

\title{
Control Chart for Failure-Censored Reliability Tests under Uncertainty Environment
}

\author{
Muhammad Aslam ${ }^{1, *} \mathbb{0}$, Nasrullah Khan ${ }^{2} \mathbb{C}$ and Mohammed Albassam ${ }^{1}$ \\ 1 Department of Statistics, Faculty of Science, King Abdulaziz University, Jeddah 21551, Saudi Arabia; \\ malbassam@kau.edu.sa \\ 2 Department of Statistics, UVAS, Lahore, Jhang Campus, Lahore 54000, Pakistani; nas_shan1@hotmail.com \\ * Correspondence: aslam_ravian@hotmail.com or magmuhammad@kau.edu.sa; Tel.: +966-59-3329841
}

Received: 11 November 2018; Accepted: 21 November 2018; Published: 2 December 2018

\begin{abstract}
Existing control charts based on failure-censored (Type-II) reliability tests were designed using classical statistics. Classical statistics was applied for the monitoring of the process when observations in the sample or the population were determined. Neutrosophic statistics (NS) are applied when there is uncertainty in the sample or population. In this paper, a control chart for failure-censored (Type-II) reliability tests was designed using NS. The design of a control chart for the Weibull distribution, which is applied when there is a lack of symmetry using neutrosophic statistics, is given. The proposed control chart was used to monitor the neutrosophic mean and neutrosophic variance, which are related to the neutrosophic scale parameter. The advantages of the proposed control chart over the existing control chart are discussed.
\end{abstract}

Keywords: neutrosophic logic; neutrosophic statistics; classical statistics; neutrosophic Weibull distribution; control chart

\section{Introduction}

During the manufacturing process, the monitoring of two variations, which can shift the process from the specified target, is an important task. The presence of only the common cause of variation does not affect the manufacturing process. Yet, the presence of a special cause of variation may cause more defects. The control chart is an effective tool, which is widely used in the industry for monitoring the production process. The control chart gives an immediate indication of when the process is shifted from the set target in the presence of a special case of variation. A timely indication is helpful for engineers to sort out the problems in the production process. Therefore, the main objectives of the control chart are to give a quick indication of the shift in the process, to reduce the number of defective products, and to maintain the high quality of the product. Control charts are designed under the assumption that the production data follow the normal distribution or come from the normal manufacturing process. However, experience show that, in the chemical industry, the processes of cutting tool wear and concentrate production follow a skewed distribution [1]. Therefore, several researchers designed control charts in the case of non-normal underlying distributions. Reference [2] designed a control chart for sign statistics. Reference [3] designed Shewhart control charts in the case of skewed data. Reference [4] presented a chart for a gamma distribution. Reference [5] developed a cost model chart for non-normal data. Reference [6] proposed a median control chart. Reference [7] proposed a chart for the Burr distribution. For more details on such control charts, the reader may refer to [8-18].

In the modern era, every reputable industry and company is trying to enhance the quality of their product. The target of creating high-quality products is achieved only by increasing the average lifetime of the product. The reliability, which is the probability that an equipment or a product performs 
well for the specified period, is used to measure the high quality of the product. For the monitoring of highly reliable products, it is not possible to wait for a specified number of failures. To save cost and time, time-truncated experiments are important. In Type-I censoring, the time of the experiment is fixed and, in Type-II censoring, the number of failures is fixed. The applications of Type-I censoring, Type-II censoring, and progressive and mixed censoring are found in [19-26].

The Weibull distribution is very popular in the area of quality control and reliability due to its flexibility of parameters. The Weibull distribution, due to its bathtub curve, is fitted well to the reliability phenomena. References [27-29] discussed the applications of the Weibull distribution. Reference [30] designed a control chart in which the process follows a Weibull distribution. Reference [31] designed a bootstrap control chart for this distribution. References [32,33] introduced control charts using time-truncated life tests. Recently, Khan et al. [34] designed a control chart for a failure-censored reliability test for the Weibull distribution. A detailed discussion of Type-II censoring control charts can be found in references [19-26].

The existing control chart using Type-II censoring is designed under the assumption that all observations are determined. According to reference [35], "observations include human judgments, and evaluations and decisions; a continuous random variable of a production process should include the variability caused by human subjectivity or measurement devices, or environmental conditions. These variability causes create vagueness in the measurement system." In this case, a control chart using the fuzzy approach was used for monitoring the process. Therefore, fuzzy logic is applied to the design of control charts when the experiment is not sure about some parameters. Several authors contributed in this area and designed control charts using the fuzzy approach such as reference [36] who introduced fuzzy logic in statistical quality control (SQC). Reference [37] introduced an algorithm using fuzzy approach. Reference [38] discussed the application of fuzzy control charts. Reference [35] proposed a Shewhart control chart using this approach. Reference [39] presented a literature review on fuzzy control charts. Reference [40] worked on a fuzzy U control chart. Reference [41] designed fuzzy variable control charts and Reference [42] also worked on a fuzzy control chart. More details on fuzzy control charts can be found in references [43-49]. Industrial applications can be found in references [50-53].

Reference [54] mentioned that traditional fuzzy logic is a special case of neutrosophic logic (NS). According to reference [54], neutrosophic logic can be applied when there exists indeterminacy in the observations or the parameters. Based on neutrosophic logic, reference [55] introduced descriptive neutrosophic statistics (NS). Reference [55] argued that NS is an extension of classical statistics. NS has many applications in a variety of areas. References $[56,57]$ applied NS to study rock roughness issues. References [58,59] designed sampling plans using NS. Recently, reference [60] introduced NS in the area of control charts. They designed an attribute control chart using the neutrosophic statistical interval method (NSIM). Reference [60] designed a variance control chart using the NSIM. They showed the efficiency of charts using NS over those based on classical statistics. Upon exploring the literature, we found no work on the design of control charts for failure-censored reliability tests in the uncertainty environment. In this paper, we focus on the design of such control charts using the NSIM. We hypothesized that the proposed chart using the NSIM would be more adequate and effective in the uncertainty environment than the existing control chart based on classical statistics. The state of the art product is described in the next section. The advantages of the proposed chart and a case study are given in Sections 3 and 4, respectively. Some conclusions are given in the final section. More details failure censored reliability can be seen in reference [61].

\section{State of the Art}

The failure time of the complements is measured through complex systems or devices. Therefore, it may be possible that some failure times are undetermined or unclear in terms of measurements. As mentioned earlier, NS is the generalization of the classical statistics that is applied when the observations in the sample are indeterminate or unclear. Suppose that the failure time indeterminacy 
interval $X_{N i} \epsilon\left\{X_{L}, X_{U}\right\}=i=1,2,3, \ldots, n_{N}$, where $X_{L}$ denotes the determinate part and $X_{U}$ denotes the indeterminate part, follows the neutrosophic Weibull distribution. Reference [59] introduced the following neutrosophic cumulative distribution function (ncdf) of the Weibull distribution.

$$
F_{N}\left(x_{N} ; m_{N}, \lambda_{N}\right)=1-\exp \left(-\left(\lambda_{N} x_{N}\right)^{m_{N}}\right) ; x_{N} \geq 0, m_{N} \epsilon\left\{m_{L}, m_{U}\right\}, \lambda_{N} \epsilon\left\{\lambda_{L}, \lambda_{U}\right\}
$$

where $m_{N} \epsilon\left\{m_{L}, m_{U}\right\}$ is the neutrosophic shape parameter and $\lambda_{N} \epsilon\left\{\lambda_{L}, \lambda_{U}\right\}$ is the neutrosophic scale parameter. The neutrosophic Weibull distribution reduces to a neutrosophic exponential distribution when $\lambda_{N} \epsilon\{-1,1\}$. The average lifetime of the neutrosophic Weibull distribution is shown below.

$$
\mu_{N}=\frac{\Gamma_{N}\left(1 / m_{N}\right)}{m_{N} \lambda_{N}} ; m_{N} \epsilon\left\{m_{L}, m_{U}\right\}, \lambda_{N} \epsilon\left\{\lambda_{L}, \lambda_{U}\right\}
$$

where $\Gamma_{N}(x)$ is the neutrosophic gamma function.

We designed a failure-censored control chart in the case of the failure time following the neutrosophic Weibull distribution to monitor the neutrosophic average and neutrosophic variance, which are related to $\lambda_{N} \epsilon\left\{\lambda_{L}, \lambda_{U}\right\}$. The proposed control chart is stated in the following steps.

1. Choose a random sample of the size $n_{N} \in\left\{n_{L}, n_{U}\right\}$ and begin the test. Continue with the test until $r_{N}$ are reached and note the ith failure time, say $X_{(i N)}\left(i=1, \ldots, r_{N}\right)$.

2. Compute the following statistic under NSIM:

$$
v_{N}=\sum_{i=1}^{r_{N}}\left(\frac{X_{(i N)}}{\mu_{0 N}}\right)^{m_{N}}+\left(n_{N}-r_{N}\right)\left(\frac{X_{(i N)}}{\mu_{0 N}}\right)^{m_{N}} ; m_{N} \epsilon\left\{m_{L}, m_{U}\right\}, \lambda_{N} \epsilon\left\{\lambda_{L}, \lambda_{U}\right\}
$$

where $\mu_{0 N} \epsilon\left\{\mu_{L}, \mu_{U}\right\}$ is the specified neutrosophic mean time.

3. Declare the process in the control state if $\mathrm{LCL}_{N} \leq v_{N} \leq \mathrm{UCL}_{N}$ where $\mathrm{LCL}_{N}$ and $\mathrm{UCL}_{N}$ denote the neutrosophic lower control limit (NLCL) and neutrosophic upper control limit (NUCL), respectively.

The operational process of the proposed control chart consists of two neutrosophic control limits. The proposed control chart under the NSIM is an extension of Khan et al. [34] control chart under the classical statistics. The proposed chart reduces to Khan et al. [34] chart when no uncertain observations or parameters are in the sample or in the population.

Suppose that the process is an in-control state at a neutrosophic scale parameter $\lambda_{0 N} \epsilon\left\{\lambda_{0 L}, \lambda_{0 U}\right\}$. The neutrosophic average life is shown in the equation below.

$$
\mu_{0 N}=\frac{\Gamma_{N}\left(1 / m_{N}\right)}{m_{N} \lambda_{N}} ; m_{N} \epsilon\left\{m_{L}, m_{U}\right\}, \lambda_{N} \epsilon\left\{\lambda_{L}, \lambda_{U}\right\}
$$

Note here that the proposed chart under NISM is independent of $\mu_{0 N} \epsilon\left\{\mu_{L}, \mu_{U}\right\}$. Reference [60] mentioned that statistic $v_{N} \in\left\{v_{L}, v_{U}\right\}$ is modeled by the neutrosophic gamma distribution with $r_{N} \in\left\{r_{L}, r_{U}\right\}$ and $w_{0 N} \in\left\{w_{0 L}, w_{0 U}\right\}$ (see [60]). The neutrosophic parameter $w_{0 N} \in\left\{w_{0 L}, w_{0 U}\right\}$ is defined by the equation below.

$$
w_{0 N}=\left(\lambda_{0 N} \mu_{0 N}\right)^{m_{N}}=\left(\frac{\Gamma_{N}\left(1 / m_{N}\right)}{m_{N}}\right)^{m_{N}} ; m_{N} \epsilon\left\{m_{L}, m_{U}\right\}, \lambda_{0 N} \epsilon\left\{\lambda_{0 L}, \lambda_{0 U}\right\}
$$

Note here that $2 v_{N} w_{0 N}$ with $2 r_{N}$ degrees of freedom is modeled by a neutrosophic chi-squared distribution. The probability that the process is an in-control state, say $\mathrm{P}_{\mathrm{in}}^{0 \mathrm{~N}}$ at $\lambda_{0 N} \epsilon\left\{\lambda_{0 L}, \lambda_{0 U}\right\}$ under the NISM, is derived by using the equation below.

$$
\mathrm{P}_{\mathrm{in}}^{0 \mathrm{~N}} \mid \lambda_{0 N}=\mathrm{P}\left(\mathrm{LCL}_{N} \leq v_{N} \leq \mathrm{UCL}_{N} \mid \lambda_{0 \mathrm{~N}}\right)=\mathrm{G}_{\mathrm{N}^{2} \mathrm{r}_{N}}\left(2 \mathrm{UCL}_{N} \mathrm{w}_{0 \mathrm{~N}}\right)-\mathrm{G}_{\mathrm{N}^{2} \mathrm{r}_{N}}\left(2 \mathrm{LCL}_{N} \mathrm{w}_{0 \mathrm{~N}}\right)
$$


where $\mathrm{G}_{\mathrm{N} 2 \mathrm{r}_{N}}(x)$ with $2 r_{N}$ represents the neutrosophic distribution function of neutrosophic chi-squared distribution. Similarly, the probability that the process is out-of-control when actually in control at $\lambda_{0 N} \epsilon\left\{\lambda_{0 L}, \lambda_{0 U}\right\}$ is given by the equation below.

$$
\mathrm{P}_{\text {out }}^{0 \mathrm{~N}} \mid \lambda_{0 N}=\mathrm{P}\left(v_{N} \geq \mathrm{UCL}_{N} \mid \lambda_{0 \mathrm{~N}}\right)+\mathrm{P}\left(v_{N} \leq \mathrm{LCL}_{N} \mid \lambda_{0 \mathrm{~N}}\right) ; \lambda_{0 N} \epsilon\left\{\lambda_{0 L}, \lambda_{0 U}\right\}
$$

or

$$
\mathrm{P}_{\mathrm{out}}^{0 \mathrm{~N}} \mid \lambda_{0 N}=1-\mathrm{G}_{2 \mathrm{r}_{N}}\left(2 \mathrm{UCL}_{N} \mathrm{w}_{0 \mathrm{~N}}\right)+\mathrm{G}_{2 \mathrm{r}_{N}}\left(2 \mathrm{LCL}_{N} \mathrm{w}_{0 \mathrm{~N}}\right) ; \lambda_{0 N} \epsilon\left\{\lambda_{0 L}, \lambda_{0 U}\right\} .
$$

The average run length under the NISM is known as the neutrosophic average run length (NARL), which is introduced by Aslam et al. [60] and given by the equation below.

$$
\mathrm{NARL}_{0 \mathrm{~N}}=\frac{1}{\mathrm{P}_{\mathrm{out}}^{0 \mathrm{~N}} \mid \lambda_{0 N}} ; \mathrm{NARL}_{0 \mathrm{~N}} \epsilon\left\{N A R L_{0 L}, N A R L_{0 U}\right\}
$$

Several special causes of variations may shift the process away from the given target. Let $\lambda_{1 N}=$ $c \lambda_{0 N} ; \lambda_{1 N} \epsilon\left\{\lambda_{0 L}, \lambda_{0 U}\right\}$ denotes the shifted neutrosophic scale parameter where $c$ denotes the shift constant. The neutrosophic average life and $w_{1 N}$ parameter at $\lambda_{1 N}$ is given by Equations (10) and (11).

$$
\begin{gathered}
\mu_{1 N}=\frac{\Gamma_{N}\left(1 / m_{N}\right)}{m_{N} \lambda_{1 N}} ; m_{N} \epsilon\left\{m_{L}, m_{U}\right\}, \lambda_{N} \epsilon\left\{\lambda_{L}, \lambda_{U}\right\} \\
w_{1 N}=\left(\lambda_{1 N} \mu_{0}\right)^{m}=c^{m} w_{0 N} .
\end{gathered}
$$

Note that $v_{N} \epsilon\left\{v_{L}, v_{U}\right\}$ is modeled by the neutrosophic gamma distribution having $r_{N} \epsilon\left\{r_{L}, r_{U}\right\}$ and $w_{1 N} \epsilon\left\{w_{1 L}, w_{1 U}\right\}$. The probability of in-control under the NISM at $\lambda_{1 N} \epsilon\left\{\lambda_{0 L}, \lambda_{0 U}\right\}$ is given by the formula below.

$$
\mathrm{P}_{\mathrm{in}}^{1 \mathrm{~N}} \mid \lambda_{1 N}=\mathrm{P}\left(\mathrm{LCL}_{N} \leq v_{N} \leq \mathrm{UCL}_{N} \mid \lambda_{1 \mathrm{~N}}\right)=\mathrm{G}_{2 \mathrm{r}_{N}}\left(2 \mathrm{UCL}_{N} \mathrm{w}_{1 \mathrm{~N}}\right)-\mathrm{G}_{2 \mathrm{r}_{N}}\left(2 \mathrm{LCL}_{N} \mathrm{w}_{1 \mathrm{~N}}\right) .
$$

The probability of out-of-control under the NISM at $\lambda_{1 N} \epsilon\left\{\lambda_{0 L}, \lambda_{0 U}\right\}$ is given by Equation (13) or Equation (14) below.

$$
\begin{aligned}
& \mathrm{P}_{\mathrm{in}}^{1 \mathrm{~N}} \mid \lambda_{1 N}=\mathrm{P}\left(v_{N} \geq \mathrm{UCL}_{N} \mid \lambda_{1 \mathrm{~N}}\right)+\mathrm{P}\left(v_{N} \leq \mathrm{LCL}_{N} \mid \lambda_{1 \mathrm{~N}}\right) \\
& \mathrm{P}_{\mathrm{in}}^{1 \mathrm{~N}} \mid \lambda_{1 N}=1-\mathrm{G}_{2 \mathrm{r}_{N}}\left(2 \mathrm{UCL}_{N} \mathrm{w}_{1 \mathrm{~N}}\right)+\mathrm{G}_{2 \mathrm{r}_{N}}\left(2 \mathrm{LCL}_{N} \mathrm{w}_{1 \mathrm{~N}}\right) .
\end{aligned}
$$

The NARL for the shifted process is given by Equation (15).

$$
\mathrm{NARL}_{1 \mathrm{~N}}=\frac{1}{\mathrm{P}_{\mathrm{out}}^{1 \mathrm{~N}} \mid \lambda_{0 N}} ; \mathrm{NARL}_{1 \mathrm{~N}} \epsilon\left\{N A R L_{1 L}, N A R L_{1 U}\right\}
$$

Suppose that $r_{0 N} \epsilon\left\{r_{L}, r_{U}\right\}$ denotes the specified value of $N_{A R L_{0 N}} \epsilon\left\{N A R L_{0 L}, N A R L_{0 U}\right\}$. The values of NARL $L_{1 \mathrm{~N}} \epsilon\left\{N A R L_{1 L}, N A R L_{1 U}\right\}$ are shown in Tables $1-4$ for various values of $m_{N} \epsilon\left\{m_{L}, m_{U}\right\}$, $\lambda_{N} \epsilon\left\{\lambda_{L}, \lambda_{U}\right\}$ and $r_{N} \epsilon\left\{r_{L}, r_{U}\right\}$. From Tables 1-4, we note that, for the same values of other specified parameters, the values of NARL decrease as the neutrophil parameter $m_{N}$ increases. 
Table 1. The values of NARL when $m_{N}=[0.4,0.6]$ and $\lambda_{N}=[0.45,0.55]$.

\begin{tabular}{|c|c|c|c|}
\hline Neutrosophic Control Limits & & $r_{N}=[2,5]$ & \\
\hline $\mathrm{LCL}_{N}$ & {$[0.0595,0.778]$} & {$[0.0484,0.708]$} & {$[0.0401,0.621]$} \\
\hline $\mathrm{UCL}_{N}$ & {$[6.1131,11.498]$} & {$[6.6267,11.801]$} & {$[6.0133,11.331]$} \\
\hline$c$ & & NARL $_{1 \mathrm{~N}}$ & \\
\hline 0.1 & {$[10.28,1.45]$} & {$[13.43,1.49]$} & {$[9.81,1.43]$} \\
\hline 0.2 & {$[28.02,2.91]$} & {$[40.55,3.11]$} & {$[26.63,2.81]$} \\
\hline 0.3 & {$[57.00,6.20]$} & {$[88.45,6.86]$} & {$[55.42,5.87]$} \\
\hline 0.4 & {$[95.77,13.22]$} & {$[155.28,15.12]$} & {$[98.58,12.32]$} \\
\hline 0.5 & {$[137.44,27.61]$} & {$[226.82,32.64]$} & {$[155.05,25.52]$} \\
\hline 0.6 & {$[172.70,55.32]$} & {$[283.97,67.72]$} & {$[218.65,51.63]$} \\
\hline 0.7 & {$[195.74,102.02]$} & {$[316.96,130.22]$} & {$[279.38,100.34]$} \\
\hline 0.75 & {$[202.48,131.52]$} & {$[324.90,172.01]$} & {$[305.64,136.51]$} \\
\hline 0.8 & {$[206.48,161.66]$} & {$[328.31,216.90]$} & {$[328.06,181.27]$} \\
\hline 0.85 & {$[208.20,188.06]$} & {$[328.22,258.76]$} & {$[346.29,233.11]$} \\
\hline 0.9 & {$[208.11,206.64]$} & {$[325.53,290.79]$} & {$[360.32,287.91]$} \\
\hline 0.92 & {$[207.68,211.35]$} & {$[323.91,299.73]$} & {$[364.80,309.24]$} \\
\hline 0.95 & {$[206.66,215.43]$} & {$[321.01,308.62]$} & {$[370.39,339.15]$} \\
\hline 0.98 & {$[205.29,216.16]$} & {$[317.68,312.24]$} & {$[374.70,365.18]$} \\
\hline 0.99 & {$[204.76,215.74]$} & {$[316.49,312.36]$} & {$[375.88,372.78]$} \\
\hline 1 & {$[204.20,215.021]$} & {$[315.26,311.99]$} & {$[376.92,379.77]$} \\
\hline 1.1 & {$[197.40,196.27]$} & {$[301.78,288.42]$} & {$[381.40,413.47]$} \\
\hline 1.2 & {$[189.38,168.95]$} & {$[287.42,248.89]$} & {$[377.72,392.50]$} \\
\hline 1.3 & {$[181.06,142.89]$} & {$[273.34,210.15]$} & {$[369.13,347.01]$} \\
\hline 1.4 & {$[172.94,120.90]$} & {$[260.06,177.26]$} & {$[357.92,298.46]$} \\
\hline 1.5 & {$[165.25,103.03]$} & {$[247.76,150.53]$} & {$[345.55,255.18]$} \\
\hline 1.6 & {$[158.07,88.61]$} & {$[236.47,129.01]$} & {$[332.90,218.90]$} \\
\hline 1.7 & {$[151.42,76.92]$} & {$[226.14,111.61]$} & {$[320.47,189.04]$} \\
\hline 1.8 & {$[145.30,67.36]$} & {$[216.70,97.42]$} & {$[308.54,164.53]$} \\
\hline 1.9 & {$[139.65,59.47]$} & {$[208.06,85.73]$} & {$[297.23,144.29]$} \\
\hline 2 & {$[134.44,52.89]$} & {$[200.13,76.01]$} & {$[286.60,127.46]$} \\
\hline 2.5 & {$[113.69,32.17]$} & {$[168.76,45.55]$} & {$[242.88,75.01]$} \\
\hline 3 & {$[99.01,21.79]$} & {$[146.74,30.45]$} & {$[211.32,49.30]$} \\
\hline 4 & {$[79.63,12.21]$} & {$[117.73,16.65]$} & {$[169.38,26.17]$} \\
\hline 5 & {$[67.30,8.05]$} & {$[99.31,10.76]$} & {$[142.69,16.46]$} \\
\hline 6 & {$[58.70,5.87]$} & {$[86.47,7.69]$} & {$[124.09,11.50]$} \\
\hline
\end{tabular}

Table 2. The values of NARL when $m_{N}=[0.9,1.10]$ and $\lambda_{N}=[0.45,0.55]$.

\begin{tabular}{cccc}
\hline Neutrosophic Control Limits & \multicolumn{3}{c}{$r_{N}=[2,5]$} \\
\hline $\mathrm{LCL}_{N}$ & {$[0.0865,1.08]$} & {$[0.0693,0.961]$} & {$[0.0621,0.933]$} \\
$\mathrm{UCL}_{N}$ & {$[8.7698,16.05]$} & {$[9.1713,16.073]$} & {$[9.2462,17.103]$} \\
\hline$c$ & & NARL $_{1 \mathrm{~N}}$ & \\
\hline 0.1 & {$[1.47,1.01]$} & {$[1.51,1.00]$} & {$[1.52,1.00]$} \\
0.2 & {$[2.73,1.14]$} & {$[2.92,1.14]$} & {$[2.96,1.12]$} \\
0.3 & {$[5.42,1.64]$} & {$[6.05,1.64]$} & {$[6.17,1.55]$} \\
0.4 & {$[11.04,2.96]$} & {$[12.83,2.97]$} & {$[13.21,2.66]$} \\
0.5 & {$[22.54,6.42]$} & {$[27.35,6.46]$} & {$[28.43,5.44]$} \\
0.6 & {$[45.01,15.99]$} & {$[57.26,16.15]$} & {$[60.31,12.76]$} \\
0.7 & {$[84.15,43.51]$} & {$[112.87,44.57]$} & {$[121.43,33.20]$} \\
0.75 & {$[109.92,71.90]$} & {$[151.64,75.13]$} & {$[165.63,54.94]$} \\
0.8 & {$[137.49,114.27]$} & {$[195.01,124.35]$} & {$[216.93,91.1923033]$} \\
0.85 & {$[163.49,165.47]$} & {$[237.85,193.61]$} & {$[270.02,148.57]$} \\
0.9 & {$[184.36,206.18]$} & {$[273.92,266.89]$} & {$[317.40,228.62]$} \\
0.92 & {$[190.70,214.58]$} & {$[285.30,289.78]$} & {$[333.15,264.14]$} \\
0.95 & {$[197.82,217.32]$} & {$[298.53,310.99]$} & {$[352.37,314.84]$} \\
\hline
\end{tabular}


Table 2. Cont.

\begin{tabular}{cccc}
\hline Neutrosophic Control Limits & \multicolumn{3}{c}{$r_{N}=[2,5]$} \\
\hline 0.98 & {$[202.13,210.10]$} & {$[307.11,314.91]$} & {$[365.94,354.61]$} \\
0.99 & {$[202.98,206.08]$} & {$[308.97,312.77]$} & {$[369.22,364.14]$} \\
1 & {$[203.55,201.49]$} & {$[310.37,309.19]$} & {$[371.89,371.54]$} \\
1.1 & {$[197.60,145.94]$} & {$[304.06,235.50]$} & {$[371.27,343.99]$} \\
1.2 & {$[180.35,101.35]$} & {$[277.91,163.84]$} & {$[342.17,250.67]$} \\
1.3 & {$[161.00,71.96]$} & {$[247.86,115.38]$} & {$[306.11,177.06]$} \\
1.4 & {$[143.14,52.65]$} & {$[220.06,83.59]$} & {$[272.03,127.50]$} \\
1.5 & {$[127.65,39.62]$} & {$[195.98,62.26]$} & {$[242.29,94.23]$} \\
1.6 & {$[114.45,30.55]$} & {$[175.50,47.53]$} & {$[216.92,71.35]$} \\
1.7 & {$[103.22,24.08]$} & {$[158.11,37.08]$} & {$[195.36,55.21]$} \\
1.8 & {$[93.64,19.35]$} & {$[143.28,29.49]$} & {$[176.96,43.55]$} \\
1.9 & {$[85.40,15.82]$} & {$[130.53,23.86]$} & {$[161.16,34.95]$} \\
2 & {$[78.26,13.13]$} & {$[119.51,19.60]$} & {$[147.48,28.49]$} \\
2.5 & {$[53.68,6.21]$} & {$[81.58,8.83]$} & {$[100.47,12.32]$} \\
3 & {$[39.61,3.67]$} & {$[59.90,4.97]$} & {$[73.63,6.67]$} \\
4 & {$[24.73,1.92]$} & {$[37.06,2.38]$} & {$[45.38,2.97]$} \\
5 & {$[17.31,1.37]$} & {$[25.72,1.58]$} & {$[31.38,1.85]$} \\
6 & {$[13.03,1.15]$} & {$[19.20,1.26]$} & {$[23.33,1.40]$} \\
\hline
\end{tabular}

Table 3. The values of NARL when $m_{N}=[2.4,2.6]$ and $\lambda_{N}=[0.45,0.55]$.

\begin{tabular}{|c|c|c|c|}
\hline \multirow{3}{*}{$\begin{array}{c}\text { Neutrosophic Control Limits } \\
\mathrm{LCL}_{N} \\
\mathrm{UCL}_{N}\end{array}$} & \multicolumn{3}{|c|}{$r_{N}=[2,5]$} \\
\hline & {$[0.119,1.27]$} & {$[0.106,1.07]$} & {$[0.0882,0.955]$} \\
\hline & {$[12.029,18.76]$} & {$[13.87,18.84]$} & {$[13.089,18.984]$} \\
\hline$c$ & \multicolumn{3}{|c|}{$\mathrm{NARL}_{1 \mathrm{~N}}$} \\
\hline 0.1 & {$[1.00,1.00]$} & {$[1.00,1.00]$} & {$[1.00,1.00]$} \\
\hline 0.2 & {$[1.0,1.00]$} & {$[1.02,1.00]$} & {$[1.01,1.00]$} \\
\hline 0.3 & {$[1.09,1.00]$} & {$[1.12,1.00]$} & {$[1.11,1.00]$} \\
\hline 0.4 & {$[1.35,1.01]$} & {$[1.47,1.01]$} & {$[1.42,1.01]$} \\
\hline 0.5 & {$[2.03,1.09]$} & {$[2.41,1.09]$} & {$[2.24,1.09]$} \\
\hline 0.6 & {$[3.85,1.44]$} & {$[5.19,1.44]$} & {$[4.57,1.45]$} \\
\hline 0.7 & {$[9.45,2.74]$} & {$[15.11,2.77]$} & {$[12.40,2.81]$} \\
\hline 0.75 & {$[16.32,4.52]$} & {$[28.77,4.59]$} & {$[22.75,4.70]$} \\
\hline 0.8 & {$[29.86,8.52]$} & {$[58.00,8.7 .00]$} & {$[44.56,8.99]$} \\
\hline 0.85 & {$[56.52,18.41]$} & {$[117.97,18.96]$} & {$[90.98,19.80]$} \\
\hline 0.9 & {$[104.00,45]$} & {$[214.7,47.4]$} & {$[180.79,50.26]$} \\
\hline 0.92 & {$[128.33,65.61]$} & {$[254.10,70.55]$} & {$[229.02,75.59]$} \\
\hline 0.95 & {$[164.80,113.08]$} & {$[295.89,129.5]$} & {$[302.41,142.57]$} \\
\hline 0.98 & {$[191.91,173.85]$} & {$[307.79,226.65]$} & {$[355.84,263.97]$} \\
\hline 0.99 & {$[197.40,191.41]$} & {$[305.72,264.90]$} & {$[366.03,317.43]$} \\
\hline 1 & {$[200.93,204.62]$} & {$[301.36,302.15]$} & {$[372.12,374.36]$} \\
\hline 1.1 & {$[166.99,127.68]$} & {$[214.78,262.21]$} & {$[300.84,411.25]$} \\
\hline 1.2 & {$[114.56,53.35]$} & {$[144.52,106.83]$} & {$[204.15,166.01]$} \\
\hline 1.3 & {$[79.65,24.85]$} & {$[100.14,47.67]$} & {$[141.14,72.39]$} \\
\hline 1.4 & {$[57.02,12.92]$} & {$[71.51,23.63]$} & {$[100.48,34.96]$} \\
\hline 1.5 & {$[41.92,7.41]$} & {$[52.44,12.87]$} & {$[73.43,18.52]$} \\
\hline 1.6 & {$[31.55,4.65]$} & {$[39.36,7.64]$} & {$[54.91,10.67]$} \\
\hline 1.7 & {$[24.26,3.16]$} & {$[30.16,4.91]$} & {$[41.91,6.63]$} \\
\hline 1.8 & {$[19.00,2.31]$} & {$[23.55,3.38]$} & {$[32.59,4.42]$} \\
\hline 1.9 & {$[15.15,1.81]$} & {$[18.70,2.49]$} & {$[25.76,3.14]$} \\
\hline 2 & {$[12.26,1.49]$} & {$[15.08,1.94]$} & {$[20.67,2.37]$} \\
\hline 2.5 & {$[5.18,1.03]$} & {$[6.23,1.08]$} & {$[8.29,1.14]$} \\
\hline 3 & {$[2.82,1.00]$} & {$[3.30,1.00]$} & {$[4.23,1.00]$} \\
\hline 4 & {$[1.40,1.00]$} & {$[1.54,1.00]$} & {$[1.82,1.00]$} \\
\hline 5 & {$[1.08,1.00]$} & {$[1.12,1.00]$} & {$[1.21,1.00]$} \\
\hline 6 & {$[1.01,1.00]$} & {$[1.02,1.00]$} & {$[1.04,1.00]$} \\
\hline
\end{tabular}


Table 4. The values of NARL when $m_{N}=[2.40,2.60]$ and $\lambda_{N}=[1.90,2.10]$.

\begin{tabular}{|c|c|c|c|}
\hline \multirow{3}{*}{$\begin{array}{c}\text { Neutrosophic Control Limits } \\
\mathrm{LCL}_{N} \\
\mathrm{UCL}_{N}\end{array}$} & \multicolumn{3}{|c|}{$r_{N}=[2,5]$} \\
\hline & {$[0.121,1.36]$} & {$[0.109,0.815]$} & {$[0.0908,1.21]$} \\
\hline & {$[12.3,19.9]$} & {$[15.135,18.198]$} & {$[13.5992,21.39]$} \\
\hline$c$ & \multicolumn{3}{|c|}{ NARL $_{1 \mathrm{~N}}$} \\
\hline 0.1 & {$[1.00,1.00]$} & {$[1.00,1.00]$} & {$[1.00,1.00]$} \\
\hline 0.2 & {$[1.01,1.00]$} & {$[1.02,1.00]$} & {$[1.02,1.00]$} \\
\hline 0.3 & {$[1.10,1.00]$} & {$[1.15,1.00]$} & {$[1.12,1.00]$} \\
\hline 0.4 & {$[1.37,1.01]$} & {$[1.55,1.00]$} & {$[1.45,1.02]$} \\
\hline 0.5 & {$[2.08,1.11]$} & {$[2.72,1.07]$} & {$[2.35,1.14]$} \\
\hline 0.6 & {$[4.02,1.53]$} & {$[6.42,1.39]$} & {$[4.97,1.68]$} \\
\hline 0.7 & {$[10.11,3.18]$} & {$[20.95,2.55]$} & {$[14.12,3.89]$} \\
\hline 0.75 & {$[17.71,5.54]$} & {$[42.43,4.10]$} & {$[26.61,7.31]$} \\
\hline 0.8 & {$[32.83,11.15]$} & {$[89.79,7.50]$} & {$[53.45,16.03]$} \\
\hline 0.85 & {$[62.65,25.8]$} & {$[181.82,15.73]$} & {$[110.80,40.93]$} \\
\hline 0.9 & {$[114.54,66.39]$} & {$[294.62,37.93]$} & {$[216.44,117.07]$} \\
\hline 0.92 & {$[140.01,96.61]$} & {$[323.83,56.07]$} & {$[268.18,176.84]$} \\
\hline 0.95 & {$[175.93,156.06]$} & {$[337.46,104.54]$} & {$[337.22,294.34]$} \\
\hline 0.98 & {$[199.53,203.24]$} & {$[322.35,200.44]$} & {$[375.37,375.25]$} \\
\hline 0.99 & {$[203.48,208.65]$} & {$[313.57,248.88]$} & {$[379.59,379.24]$} \\
\hline 1 & {$[205.45,208.09]$} & {$[303.79,307.56]$} & {$[379.96,371.89]$} \\
\hline 1.1 & {$[163.83,98.63]$} & {$[203.67,744.86]$} & {$[286.61,161.48]$} \\
\hline 1.2 & {$[111.83,41.49]$} & {$[136.53,321.09]$} & {$[193.27,65.86]$} \\
\hline 1.3 & {$[77.73,19.68]$} & {$[94.63,136.05]$} & {$[133.63,30.25]$} \\
\hline 1.4 & {$[55.66,10.43]$} & {$[67.61,63.56]$} & {$[95.17,15.48]$} \\
\hline 1.5 & {$[40.93,6.11]$} & {$[49.61,32.46]$} & {$[69.59,8.73]$} \\
\hline 1.6 & {$[30.82,3.92]$} & {$[37.26,17.98]$} & {$[52.07,5.38]$} \\
\hline 1.7 & {$[23.70,2.73]$} & {$[28.58,10.71]$} & {$[39.77,3.59]$} \\
\hline 1.8 & {$[18.58,2.04]$} & {$[22.33,6.83]$} & {$[30.94,2.58]$} \\
\hline 1.9 & {$[14.81,1.63]$} & {$[17.75,4.64]$} & {$[24.47,1.98]$} \\
\hline 2 & {$[12.00,1.38]$} & {$[14.33,3.34]$} & {$[19.65,1.61]$} \\
\hline 2.5 & {$[5.08,1.02]$} & {$[5.95,1.29]$} & {$[7.92,1.04]$} \\
\hline 3 & {$[2.77,1.00]$} & {$[3.17,1.02]$} & {$[4.06,1.00]$} \\
\hline 4 & {$[1.39,1.00]$} & {$[1.51,1.00]$} & {$[1.77,1.00]$} \\
\hline 5 & {$[1.07,1.00]$} & {$[1.11,1.00]$} & {$[1.19,1.00]$} \\
\hline 6 & {$[1.01,1.00]$} & {$[1.01,1.00]$} & {$[1.04,1.00]$} \\
\hline
\end{tabular}

The following algorithm under the NISM is applied to determine the neutrosophic control limits and NARL ${ }_{1 N} \in\left\{N A R L_{1 L}, N A R L_{1 U}\right\}$ :

1. Specify $m_{N} \in\left\{m_{L}, m_{U}\right\}, \lambda_{N} \epsilon\left\{\lambda_{L}, \lambda_{U}\right\}$ and $r_{N} \epsilon\left\{r_{L}, r_{U}\right\}$.

2. Specify $r_{0 N} \in\left\{r_{L}, r_{U}\right\}$ and determine the neutrosophic control limits such that $\mathrm{NARL}_{0 \mathrm{~N}} \geq r_{0 N}$.

3. Several combinations exist that satisfy the condition $N A R L_{0 N} \geq r_{0 N}$. However, choose the combination of the neutrosophic parameters where NARL $\mathrm{N}_{0 \mathrm{~N}}$ is very close to $r_{0 N}$.

4. Use neutrosophic control limits to find $N A R L_{1 N} \epsilon\left\{N A R L_{1 L}, N A R L_{1 U}\right\}$.

\section{Advantages of the Proposed Chart}

In the NS, by following reference [54], we defined the NARL as NARL $L_{1 \mathrm{~N}}=A R L_{1 L}+u I$ where $A R L_{1 L}$ is the average run length (ARL) for the determined part under the classical statistics, $u I$ is an indeterminate part for $A R L_{1 L}$, and I $\epsilon\{$ infI, supI $\}$ presents the indeterminacy. When there are certain observations in the sample or in the population, the indeterminate part $u I=0$ and NARL $1 \mathrm{~N}$ under the NS becomes the same as the ARL under the classical statistics. According to reference [57], under the uncertainty settings, a method that provides the indeterminacy interval of NARL is said to be a more efficient and effective method than the method that provides a determined value of $A R L_{1}$. The values of NARL ${ }_{1 N}$ from the proposed control chart under NISM and ARL $L_{1}$ from Aslam et al. [34] under the 
classical statistics are in Table 5 at the same levels of all specified control chart parameters. From Table 5, we note that the proposed control chart has the values of NARL $1 \mathrm{~N}$ in the indeterminacy interval while the existing control chart under the classical statistics provides only the determined values of $\mathrm{ARL}_{1}$. For example, when $c=0.1$, we have NARL $1 \mathrm{~N}=1.45+1.45 I ; I \epsilon\{0,7.09\}$. Thus, the determinate par is $A_{R L}$ $=1.45$ and the indeterminate part is $1.45 I ; I \epsilon\{0,7.09\}$. Therefore, the indeterminacy interval of NARL $1 \mathrm{~N}$ is $\mathrm{NARL}_{1 \mathrm{~N}}=[10.287,1.451] ; I \epsilon\{0,7.09\}$. From this example, it is clear that the proposed control chart has determinate and indeterminate information under the indeterminate situation. Therefore, the proposed control chart is more effective under the indeterminate situation than Aslam et al. [34] chart, which is a special case of the proposed chart.

Table 5. The comparison of the proposed chart with the existing one when $m_{N}=[0.4,0.6], \lambda_{N}=$ $[0.45,0.55]$, and $r_{N}=[2,5]$.

\begin{tabular}{|c|c|c|c|c|c|c|}
\hline \multirow{3}{*}{$\begin{array}{c}\text { Neutrosophic } \\
\text { Control Limits }\end{array}$} & \multicolumn{3}{|c|}{ Proposed Control Chart } & \multicolumn{3}{|c|}{ Existing Control Chart } \\
\hline & \multirow{2}{*}{$\mathrm{NARL}_{0 \mathrm{~N}} \in[200,200]$} & \multirow{2}{*}{ NARL $_{0 N} \in[300,300]$} & \multirow{2}{*}{$\mathrm{NARL}_{0 \mathrm{~N}} \in[370,370]$} & \multirow{2}{*}{$A R L=200$} & \multirow{2}{*}{$\mathrm{ARL}=300$} & \multirow{2}{*}{$\mathrm{ARL}=370$} \\
\hline & & & & & & \\
\hline$c$ & & NARL $_{1}$ & & & $\mathrm{ARL}_{1}$ & \\
\hline 0.1 & {$[10.28,1.45]$} & {$[13.43,1.49]$} & {$[9.81,1.43]$} & 1.45 & 1.49 & 1.43 \\
\hline 0.2 & {$[28.02,2.91]$} & {$[40.55,3.11]$} & {$[26.63,2.81]$} & 2.91 & 3.11 & 2.81 \\
\hline 0.3 & {$[57.00,6.20]$} & {$[88.45,6.86]$} & {$[55.42,5.87]$} & 6.20 & 6.86 & 5.87 \\
\hline 0.4 & {$[95.77,13.22]$} & {$[155.28,15.12]$} & {$[98.58,12.32]$} & 13.22 & 15.1 & 12.32 \\
\hline 0.5 & {$[137.44,27.61]$} & {$[226.82,32.64]$} & {$[155.05,25.52]$} & 27.61 & 32.6 & 25.52 \\
\hline 0.6 & {$[172.70,55.32]$} & {$[283.97,67.72]$} & {$[218.65,51.63]$} & 55.32 & 67.72 & 51.63 \\
\hline 0.7 & {$[195.74,102.02]$} & {$[316.96,130.22]$} & {$[279.38,100.34]$} & 102.02 & 130.22 & 100.34 \\
\hline 0.75 & {$[202.48,131.52]$} & {$[324.90,172.01]$} & {$[305.64,136.51]$} & 131.52 & 172.01 & 136.51 \\
\hline 0.8 & {$[206.48,161.66]$} & {$[328.31,216.90]$} & {$[328.06,181.27]$} & 161.66 & 216.90 & 181.27 \\
\hline 0.85 & {$[208.20,188.06]$} & {$[328.22,258.76]$} & {$[346.29,233.11]$} & 188.06 & 258.76 & 233.11 \\
\hline 0.9 & {$[208.11,206.64]$} & {$[325.53,290.79]$} & {$[360.32,287.91]$} & 206.64 & 290.79 & 287.91 \\
\hline 0.92 & {$[207.68,211.35]$} & [323.91, 299.73] & {$[364.80,309.24]$} & 211.35 & 299.73 & 309.24 \\
\hline 0.95 & {$[206.66,215.43]$} & {$[321.01,308.62]$} & {$[370.39,339.15]$} & 215.43 & 308.62 & 339.15 \\
\hline 0.98 & {$[205.29,216.16]$} & {$[317.68,312.24]$} & {$[374.70,365.18]$} & 216.16 & 312.24 & 365.18 \\
\hline 0.99 & {$[204.76,215.74]$} & {$[316.49,312.36]$} & {$[375.88,372.78]$} & 215.74 & 312.36 & 372.78 \\
\hline 1 & {$[204.20,215.02]$} & {$[315.26,311.99]$} & {$[376.92,379.77]$} & 215.02 & 311.99 & 376.92 \\
\hline 1.1 & {$[197.40,196.27]$} & {$[301.78,288.42]$} & {$[381.40,413.47]$} & 196.27 & 288.42 & 381.40 \\
\hline 1.2 & {$[189.38,168.95]$} & {$[287.42,248.89]$} & {$[377.72,392.50]$} & 168.95 & 248.89 & 377.7 \\
\hline 1.3 & {$[181.06,142.89]$} & {$[273.34,210.15]$} & {$[369.13,347.01]$} & 142.89 & 210.15 & 347.01 \\
\hline 1.4 & {$[172.94,120.90]$} & {$[260.06,177.26]$} & {$[357.92,298.46]$} & 120.90 & 177.26 & 298.46 \\
\hline 1.5 & {$[165.25,103.03]$} & {$[247.76,150.53]$} & {$[345.55,255.18]$} & 103.03 & 150.53 & 255.18 \\
\hline 1.6 & {$[158.07,88.61]$} & {$[236.47,129.01]$} & {$[332.90,218.90]$} & 88.61 & 129.01 & 218.90 \\
\hline 1.7 & {$[151.42,76.92]$} & {$[226.14,111.61]$} & {$[320.47,189.04]$} & 76.92 & 111.61 & 189.04 \\
\hline 1.8 & {$[145.30,67.36]$} & {$[216.70,97.42]$} & {$[308.54,164.53]$} & 67.36 & 97.42 & 164.53 \\
\hline 1.9 & {$[139.65,59.47]$} & {$[208.06,85.73]$} & {$[297.23,144.29]$} & 59.47 & 85.73 & 144.29 \\
\hline 2 & {$[134.44,52.89]$} & {$[200.13,76.01]$} & {$[286.60,127.46]$} & 52.89 & 76.01 & 127.46 \\
\hline 2.5 & {$[113.69,32.17]$} & {$[168.76,45.55]$} & {$[242.88,75.01]$} & 32.17 & 45.55 & 75.01 \\
\hline 3 & {$[99.01,21.79]$} & {$[146.74,30.45]$} & {$[211.32,49.30]$} & 21.79 & 30.45 & 49.30 \\
\hline 4 & {$[79.63,12.21]$} & {$[117.73,16.65]$} & {$[169.38,26.17]$} & 12.21 & 16.65 & 26.17 \\
\hline 5 & {$[67.30,8.05]$} & {$[99.31,10.76]$} & {$[142.69,16.46]$} & 8.05 & 10.76 & 16.46 \\
\hline 6 & {$[58.70,5.87]$} & {$[86.47,7.69]$} & {$[124.09,11.50]$} & 5.87 & 7.69 & 11.50 \\
\hline
\end{tabular}

Now, we compare the performance of the proposed control chart under NISM with the chart under the classical statistics using the simulated data. The neutrosophic data was generated from the neutrosophic Weibull distribution with parameters $m_{N}=[2.40,2.60]$ and $\lambda_{N}=[0.45,0.55]$. The first 20 neutrosophic observations were generated when the process was in-control at $\lambda_{N}=[0.45,0.55]$ and the next 20 neutrosophic observations were generated from the shifted process when $c=0.85$. From Table 3 , the indeterminacy interval of $\mathrm{NARL}_{1 \mathrm{~N}}$ is $\mathrm{NARL}_{1 \mathrm{~N}} \in\{90.98,19.80\}$. Under the uncertainty environment, it is expected that the process will be out-of-control between the 19th and 90th samples when $c=0.85$. The values of $v_{N} \epsilon\left\{v_{L}, v_{U}\right\}$ are plotted on the control chart in Figure 1. From Figure 1 , we note the proposed control chart under NISM detected a shift at the 39th sample. The values of $v$ are also plotted on the existing chart in Figure 2. From Figure 2, we note that the existing control chart under classical statistics did not detect any shift in the process. By comparing Figures 1 with 2, we 
conclude that the proposed control chart is more efficient in detecting an early shift in the process than the existing chart under classical statistics.

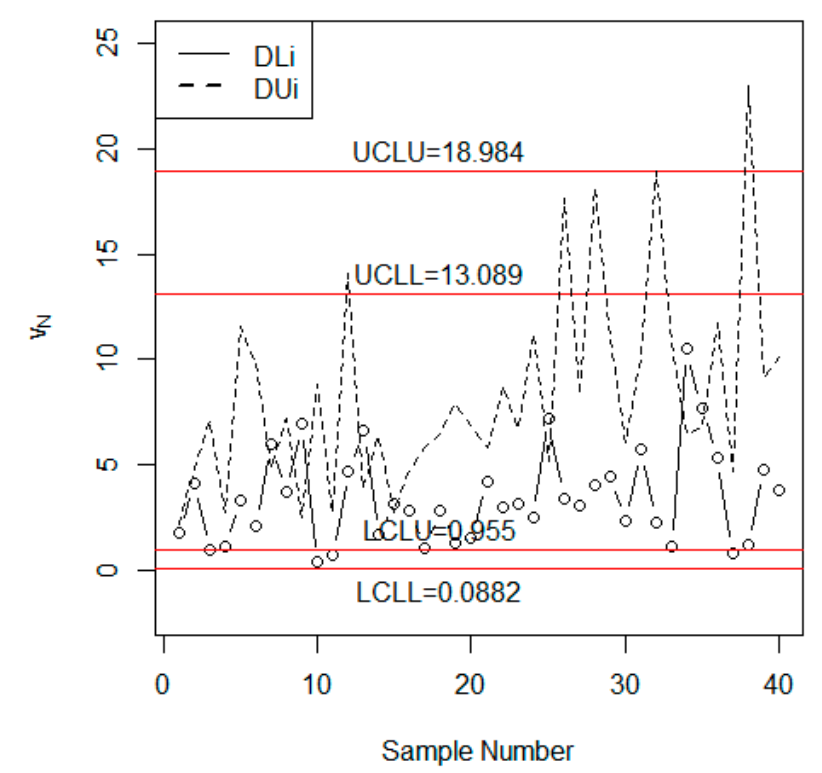

Figure 1. The proposed control chart for the simulated data.

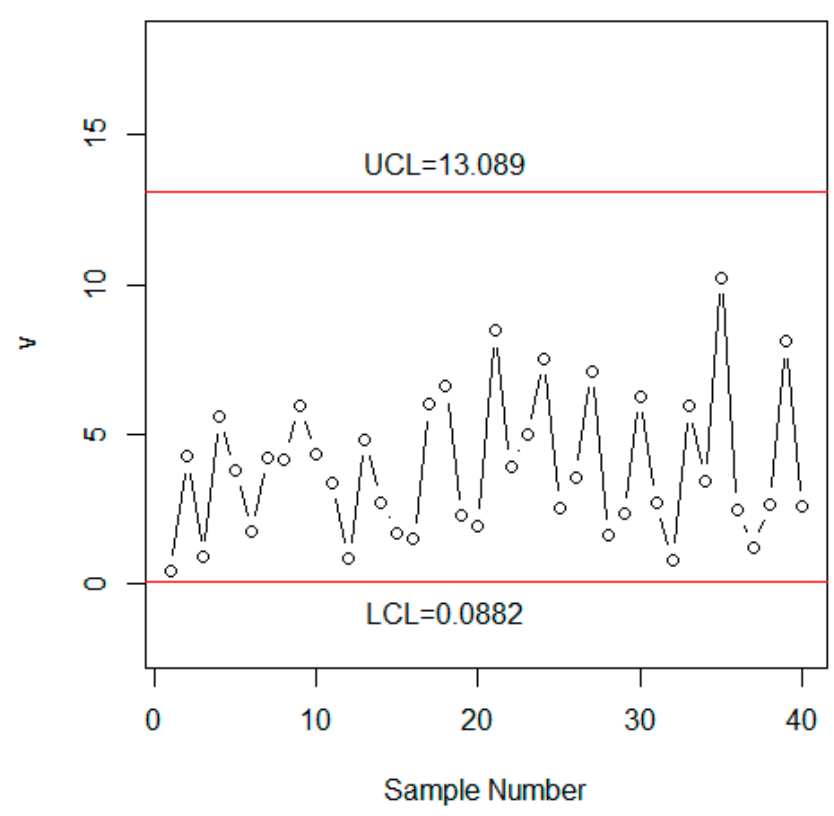

Figure 2. Aslam et al. [34] control chart for the simulated data.

\section{Case Study}

This section is presented to explain the application of the proposed control chart in the very popular automobile manufacturing industry located in Japan. For the high quality of the subsystems of the passenger's cars, the monitoring of the process is done through the control chart. The quality of the subsystems of the cars is based on the service time. The data is in days until the service is required for the subsystems. The service time data of subsystems is measured through the complex equipment. Therefore, some observations about the time until the service is required are uncertain. The monitoring of such data cannot be done using the control chart proposed by Aslam et al. [34] under classical statistics. Under the uncertainty environment, the company has decided to apply the proposed control chart for the monitoring of service time data. Suppose, for this experiment, the company decided to 
set parameters as $r_{N} \epsilon[2,5]$ and $\mathrm{NARL}_{0 \mathrm{~N}} \epsilon\{370,370\}$. The service time data of presenter cars follows the neutrosophic Weibull distribution with $m_{N} \epsilon[2.40,2.60]$ and $\lambda_{N} \epsilon[1.90,2.10]$. The $v_{N}$ for this data is shown in Table 6.

Table 6. The statistics for the service time data.

\begin{tabular}{|c|c|c|c|}
\hline Sample No. & $v_{N}$ & Sample No. & $v_{N}$ \\
\hline 1 & {$[3.01,2.76]$} & 21 & {$[0.36,16.32]$} \\
\hline 2 & {$[2.61,7.57]$} & 22 & {$[0.39,4.44]$} \\
\hline 3 & {$[7.74,4.57]$} & 23 & {$[0.98,10.49]$} \\
\hline 4 & {$[0.84,4.25]$} & 24 & {$[6.06,6.49]$} \\
\hline 5 & {$[1.19,10.66]$} & 25 & {$[7.31,2.59]$} \\
\hline 6 & {$[2.55,10.25]$} & 26 & {$[2.54,9.34]$} \\
\hline 7 & {$[3.73,7.66]$} & 27 & {$[6.18,11.58]$} \\
\hline 8 & {$[3.37,6.82]$} & 28 & {$[1.78,6.91]$} \\
\hline 9 & {$[6.19,5.43]$} & 29 & {$[2.61,7.28]$} \\
\hline 10 & {$[6.12,4.31]$} & 30 & {$[5.05,4.73]$} \\
\hline 11 & {$[3.03,9.71]$} & 31 & {$[3.67,8.89]$} \\
\hline 12 & {$[0.33,2.64]$} & 32 & {$[4.74,6.45]$} \\
\hline 13 & {$[3.46,5.18]$} & 33 & {$[0.36,13.32]$} \\
\hline 14 & {$[3.11,11.91]$} & 34 & {$[3.00,9.29]$} \\
\hline 15 & {$[1.67,5.78]$} & 35 & {$[0.67,11.71]$} \\
\hline 16 & {$[1.09,4.59]$} & 36 & {$[0.61,3.30]$} \\
\hline 17 & {$[2.29,8.98]$} & 37 & {$[2.01,1.69]$} \\
\hline 18 & {$[2.47,4.16]$} & 38 & {$[0.29,15.05]$} \\
\hline 19 & {$[1.22,11.02]$} & 39 & {$[1.00,12.85]$} \\
\hline 20 & {$[3.13,7.45]$} & 40 & {$[2.55,9.40]$} \\
\hline
\end{tabular}

For example, for sample \#1: the service time in days is $X_{N} \in\{0.357262,0.510949 ; 0.077456,0.122034$, $0.241257,0.289738,0.369004\}$. The statistic $v_{N}$ for this sample is computed by using the formula below. $v_{N} \epsilon \sum_{i=1}^{r_{N}}\left(\frac{X_{(i N)}}{\mu_{0 N}}\right)^{m_{N}}+\left(n_{N}-r_{N}\right)\left(\frac{X_{(i N)}}{\mu_{0 N}}\right)^{m_{N}}=\{2.76,3.01\} ; m_{N}=[2.40,2.60], r_{N} \epsilon[2,5]$ and $\lambda_{N}=$ $[1.90,2.10]$. The values of $v_{N}$ are plotted on the control chart in Figure 3. From Figure 3, we note that several values of the statistic lie in the indeterminacy interval of the control limits. Yet, the control chart proposed by Aslam et al. [34] in Figure 4 shows that the process is an in-control state. From Figure 3, it can be noted that several values of plotting statistics are in the indeterminacy interval. Samples \#12, $\# 21$, \#22, \#33, and \#37 are very close to the $\mathrm{LCL}_{\mathrm{L}}$ that needs special attention by the industrial engineers. However, the existing control chart indicates that only two values are very close to the control limit. By comparing Figures 3 with 4, we conclude that the proposed control chart is better, more flexible, and more effective than the existing chart under the uncertainty environment. In addition, the proposed control chart is more efficient for the monitoring of the process than the existing control chart. 


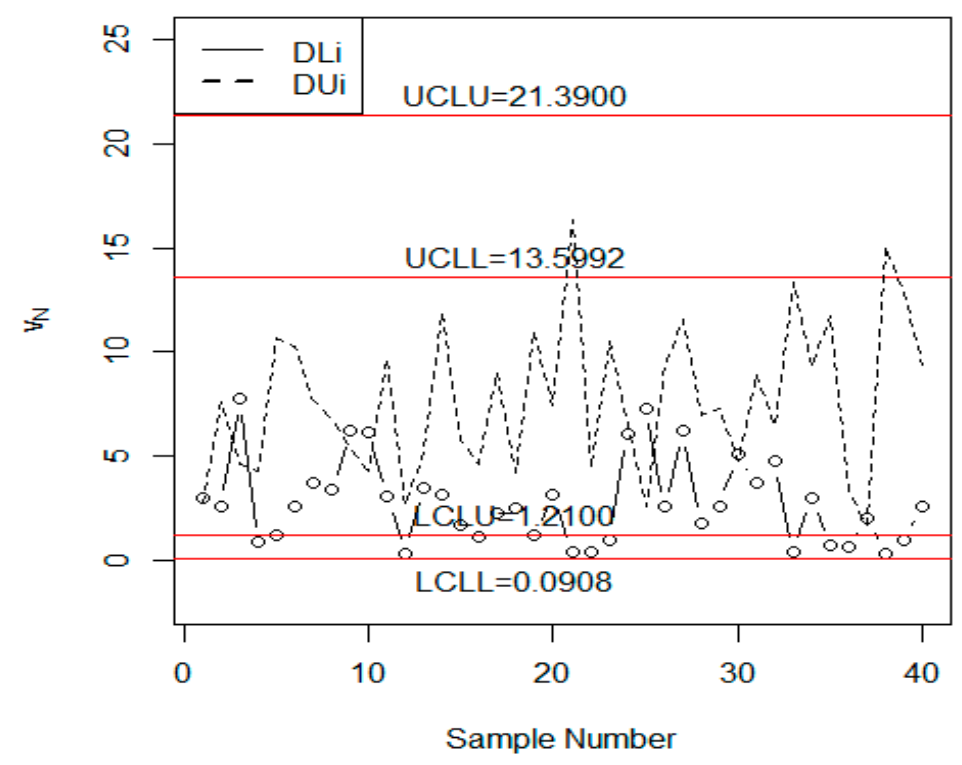

Figure 3. The proposed control chart for the automobile data.

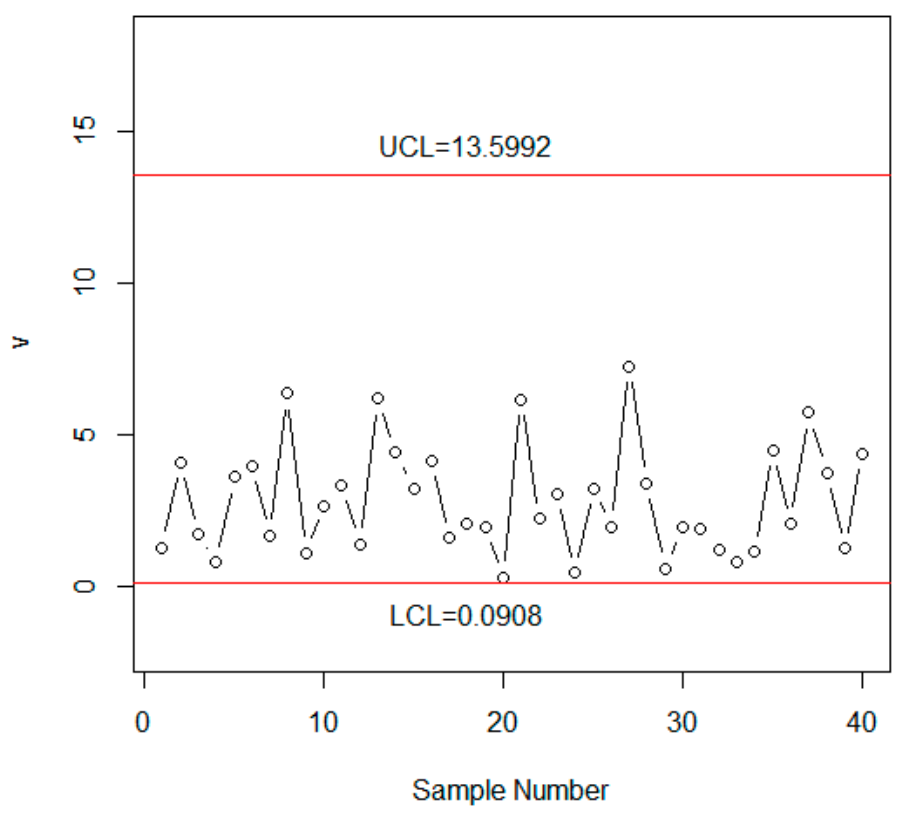

Figure 4. Aslam et al. [34] control chart for the automobile data.

\section{Conclusions}

A control chart for failure-censored (Type-II) reliability tests under the NS was presented in this paper. The necessary measures to apply the proposed control chart were given in the paper. The proposed control chart was the generalization of the control chart under classical statistics. A simulation study and real data showed the efficiency of the proposed control chart under the uncertainty environment. By comparing the proposed chart with the chart under the classical statistics, we noted that the proposed chart was more effective, more flexible, and adequate for use in the uncertainty environment. The proposed control chart can be applied in this industry when there are some uncertain, unclear, and fuzzy observations in the sample or in the population. The proposed control chart, when using the other sampling schemes or the cost model, can be studied in future research. 
Author Contributions: Conceived and designed the experiments, M.A. (Mohammed Albassam), N.K., and M.A. (Mohammed Albassam) Performed the experiments, M.A. (Mohammed Albassam) and N.K. Analyzed the data, M.A. (Mohammed Albassam) and N.K. Contributed reagents/materials/analysis tools, M.A. (Muhammad Aslam). Wrote the paper, M.A. (Muhammad Aslam).

Funding: This article was funded by the Deanship of Scientific Research (DSR) at King Abdulaziz University, Jeddah. The authors, therefore, acknowledge and thank DSR technical and financial support.

Acknowledgments: The authors are deeply thankful to the editor and reviewers for their valuable suggestions to improve the quality of this manuscript.

Conflicts of Interest: The authors declare no conflict of interest regarding this paper.

\section{References}

1. Aichouni, M.; Al-Ghonamy, A.; Bachioua, L. Control charts for non-normal data: Illustrative example from the construction industry business. Available online: https:/ / www.google.com.tw/url?sa=t\&rct=j\&q= \&esrc=s\&source=web\&cd=1\&cad=rja\&uact=8\&ved=2ahUKEwjgz4mTn_zeAhWOFogKHXjbCXIQFjAAe gQICRAC\&url=http\%3A\%2F\%2Fwww.wseas.us\%2Fe-library \%2Fconferences $\% 2 F 2014 \% 2 F M a l a y s i a \% 2 F M$ ACMESE\%2FMACMESE-10.pdf\&usg=AOvVaw1PPU0bV-aBbm7pVZ9wunqY (accessed on 11 November 2018).

2. Amin, R.W.; Reynolds, M.R., Jr.; Saad, B. Nonparametric quality control charts based on the sign statistic. Commun. Stat. Theory Methods 1995, 24, 1597-1623. [CrossRef]

3. Bai, D.; Choi, I. (x) over-bar-control and r-control charts for skewed populations. J. Qual. Technol. 1995, 27, 120-131. [CrossRef]

4. Al-Oraini, H.A.; Rahim, M. Economic statistical design of $\bar{x}$ control charts for systems with gamma $(\lambda, 2)$ in-control times. Comput. Ind. Eng. 2002, 43, 645-654. [CrossRef]

5. Chen, Y.-K. Economic design of $\mathrm{x}$ control charts for non-normal data using variable sampling policy. Int. J. Prod. Econ. 2004, 92, 61-74. [CrossRef]

6. Ahmad, S.; Riaz, M.; Abbasi, S.A.; Lin, Z. On efficient median control charting. J. Chin. Inst. Eng. 2014, 37, 358-375. [CrossRef]

7. Lio, Y.; Tsai, T.-R.; Aslam, M.; Jiang, N. Control charts for monitoring burr type-x percentiles. Commun. Stat. Simul. Comput. 2014, 43, 761-776. [CrossRef]

8. Miller, T.; Balch, B. Statistical process control in food processing. ISA Trans. 1991, 30, 35-37. [CrossRef]

9. Kegel, T. Statistical control of a pressure instrument calibration process. ISA Trans. 1996, 35, 69-77. [CrossRef]

10. Chou, C.-Y.; Chen, C.-H.; Liu, H.-R. Economic-statistical design of $x ¥$ charts for non-normal data by considering quality loss. J. Appl. Stat. 2000, 27, 939-951. [CrossRef]

11. Wu, Z.; Xie, M.; Tian, Y. Optimization design of thex \& s charts for monitoring process capability. J. Manuf. Syst. 2002, 21, 83-92.

12. Venkatesan, G. Process control of product quality. ISA Trans. 2003, 42, 631-641. [CrossRef]

13. Lin, Y.-C.; Chou, C.-Y. On the design of variable sample size and sampling intervals charts under non-normality. Int. J. Prod. Econ. 2005, 96, 249-261. [CrossRef]

14. Zhang, H.Y.; Shamsuzzaman, M.; Xie, M.; Goh, T.N. Design and application of exponential chart for monitoring time-between-events data under random process shift. Int. J. Adv. Manuf. Technol. 2011, 57, 849-857. [CrossRef]

15. McCracken, A.; Chakraborti, S. Control charts for joint monitoring of mean and variance: An overview. Qual. Technol. Quant. Manag. 2013, 10, 17-35. [CrossRef]

16. Addeh, J.; Ebrahimzadeh, A.; Azarbad, M.; Ranaee, V. Statistical process control using optimized neural networks: A case study. ISA Trans. 2014, 53, 1489-1499. [CrossRef] [PubMed]

17. Celano, G.; Castagliola, P.; Fichera, S.; Nenes, G. Performance of $\mathrm{t}$ control charts in short runs with unknown shift sizes. Comput. Ind. Eng. 2013, 64, 56-68. [CrossRef]

18. Aslam, M.; Azam, M.; Khan, N. X-bar control charts for non-normal correlated data under repetitive sampling. J. Test. Eval. 2015, 44, 1756-1767. [CrossRef]

19. Pascual, F.; Li, S. Monitoring the weibull shape parameter by control charts for the sample range of type ii censored data. Qual. Reliab. Eng. Int. 2012, 28, 233-246. [CrossRef] 
20. Guo, B.; Wang, B.X. Control charts for monitoring the weibull shape parameter based on type-ii censored sample. Qual. Reliab. Eng. Int. 2014, 30, 13-24. [CrossRef]

21. Haghighi, F.; Pascual, F.; Castagliola, P. Conditional control charts for weibull quantiles under type ii censoring. Qual. Reliab. Eng. Int. 2015, 31, 1649-1664. [CrossRef]

22. Chan, Y.; Han, B.; Pascual, F. Monitoring the weibull shape parameter with type ii censored data. Qual. Reliab. Eng. Int. 2015, 31, 741-760. [CrossRef]

23. Wang, F.K.; Bizuneh, B.; Cheng, X.B. New control charts for monitoring the weibull percentiles under complete data and type-ii censoring. Qual. Reliab. Eng. Int. 2018, 34, 403-416. [CrossRef]

24. Asadzadeh, S.; Kiadaliry, F. Monitoring type-2 censored reliability data in multistage processes. Qual. Reliab. Eng. Int. 2017, 33, 2551-2561. [CrossRef]

25. Aslam, M.; Arif, O.H.; Jun, C.-H. An attribute control chart for a weibull distribution under accelerated hybrid censoring. PLoS ONE 2017, 12, e0173406. [CrossRef] [PubMed]

26. Guo, B.; Wang, B.X.; Xie, M. Arl-unbiased control charts for the monitoring of exponentially distributed characteristics based on type-ii censored samples. J. Stat. Comput. Simul. 2014, 84, 2734-2747. [CrossRef]

27. Montgomery, D.C.; Runger, G.C. Applied Statistics and Probability for Engineers; John Wiley \& Sons: New York, NY, USA, 2010.

28. Rausand, M.; Høyland, A. System Reliability Theory: Models, Statistical Methods, and Applications; John Wiley \& Sons: New York, NY, USA, 2004; Volume 396.

29. Borror, C.M.; Keats, J.B.; Montgomery, D.C. Robustness of the time between events cusum. Int. J. Prod. Res. 2003, 41, 3435-3444. [CrossRef]

30. Nelson, P.R. Control charts for weibull processes with standards given. IEEE Trans. Reliab. 1979, 28, $283-288$. [CrossRef]

31. Nichols, M.D.; Padgett, W. A bootstrap control chart for weibull percentiles. Qual. Reliab. Eng. Int. 2006, 22, 141-151. [CrossRef]

32. Aslam, M.; Jun, C.-H. Attribute control charts for the weibull distribution under truncated life tests. Qual. Eng. 2015, 27, 283-288. [CrossRef]

33. Aslam, M.; Khan, N.; Jun, C.-H. A control chart for time truncated life tests using pareto distribution of second kind. J. Stat. Comput. Simul. 2015, 86, 2113-2122. [CrossRef]

34. Khan, N.; Aslam, M.; Raza, S.M.M.; Jun, C.H. A new variable control chart under failure-censored reliability tests for Weibull distribution. Qual. Reliab. Eng. Int. 2018. [CrossRef]

35. Senturk, S.; Erginel, N. Development of fuzzy $\bar{x} \sim-r \sim$ and $\bar{x} \sim-s \sim$ control charts using $\alpha$-cuts. Inf. Sci. 2009, 179, 1542-1551. [CrossRef]

36. Rowlands, H.; Wang, L.R. An approach of fuzzy logic evaluation and control in spc. Qual. Reliab. Eng. Int. 2000, 16, 91-98. [CrossRef]

37. El-Shal, S.M.; Morris, A.S. A fuzzy rule-based algorithm to improve the performance of statistical process control in quality systems. J. Intell. Fuzzy Syst. 2000, 9, 207-223.

38. Ertuğrul, I.; Güneş, M. The usage of fuzzy quality control charts to evaluate product quality and an application. In Analysis and Design of Intelligent Systems Using Soft Computing Techniques; Springer: Berlin/Heidelberg, Germany, 2007; pp. 660-673.

39. Sabegh, M.H.Z.; Mirzazadeh, A.; Salehian, S.; Weber, G.-W. A literature review on the fuzzy control chart; classifications \& analysis. Int. J. Supply Oper. Manag. 2014, 1, 167-189.

40. Avakh Darestani, S.; Moradi Tadi, A.; Taheri, S.; Raeiszadeh, M. Development of fuzzy u control chart for monitoring defects. Int. J. Qual. Reliab. Manag. 2014, 31, 811-821. [CrossRef]

41. Mojtaba Zabihinpour, S.; Ariffin, M.; Tang, S.H.; Azfanizam, A. Construction of fuzzy x̄s control charts with an unbiased estimation of standard deviation for a triangular fuzzy random variable. J. Intell. Fuzzy Syst. 2015, 28, 2735-2747. [CrossRef]

42. Shu, M.-H.; Dang, D.-C.; Nguyen, T.-L.; Hsu, B.-M.; Phan, N.-S. Fuzzy and control charts: A data-adaptability and human-acceptance approach. Complexity 2017, 2017, 4376809. [CrossRef]

43. Gülbay, M.; Kahraman, C.; Ruan, D. A-cut fuzzy control charts for linguistic data. Int. J. Intell. Syst. 2004, 19, 1173-1195. [CrossRef]

44. Kaya, İ.; Kahraman, C. Process capability analyses based on fuzzy measurements and fuzzy control charts. Expert Syst. Appl. 2011, 38, 3172-3184. [CrossRef] 
45. Soleymani, P.; Amiri, A. Fuzzy cause selecting control chart for monitoring multistage processes. Int. J. Ind. Syst. Eng. 2017, 25, 404-422. [CrossRef]

46. Ercan Teksen, H.; Anagun, A.S. Different methods to fuzzy $\bar{x}-r$ control charts used in production: Interval type-2 fuzzy set example. J. Enterp. Inf. Manag. 2018, 31, 848-866. [CrossRef]

47. Alakoc, N.P.; Apaydin, A. A fuzzy control chart approach for attributes and variables. Eng. Technol. Appl. Sci. Res. 2018, 8, 3360-3365.

48. Mashuri, M.; Ahsan, M. Perfomance Fuzzy Multinomial Control Chart. J. Phys. Conf. Ser. 2018, 1028, 012120.

49. Fadaei, S.; Pooya, A. Fuzzy u control chart based on fuzzy rules and evaluating its performance using fuzzy oc curve. TQM J. 2018, 30, 232-247. [CrossRef]

50. Brunetto, P.; Fortuna, L.; Giannone, P.; Graziani, S.; Strazzeri, S. Static and dynamic characterization of the temperature and humidity influence on ipmc actuators. IEEE Trans. Instrum. Meas. 2010, 59, 893-908. [CrossRef]

51. Meza, J.; Espitia, H.; Montenegro, C.; Crespo, R.G. Statistical analysis of a multi-objective optimization algorithm based on a model of particles with vorticity behavior. Soft Comput. 2016, 20, 3521-3536. [CrossRef]

52. Arora, S.; Singh, S. An effective hybrid butterfly optimization algorithm with artificial bee colony for numerical optimization. Int. J. Interact. Multimedia Artif. Intell. 2017, 4, 14-21. [CrossRef]

53. Padilla, V.; Conklin, D. Generation of Two-Voice Imitative Counterpoint from Statistical Models. Int. J. Interact. Available online: https:/ / www.ijimai.org/journal/node/2649 (accessed on 10 November 2018).

54. Smarandache, F. Neutrosophic logic-a generalization of the intuitionistic fuzzy logic. Multispace Multistruct. Neutrosophic Transdiscipl. 2010, 4, 396. [CrossRef]

55. Smarandache, F. Introduction to Neutrosophic Statistics; Infinite Study; Sitech Education Publishing: Craiova, Romania, 2014.

56. Chen, J.; Ye, J.; Du, S. Scale effect and anisotropy analyzed for neutrosophic numbers of rock joint roughness coefficient based on neutrosophic statistics. Symmetry 2017, 9, 208. [CrossRef]

57. Chen, J.; Ye, J.; Du, S.; Yong, R. Expressions of rock joint roughness coefficient using neutrosophic interval statistical numbers. Symmetry 2017, 9, 123. [CrossRef]

58. Aslam, M. A new sampling plan using neutrosophic process loss consideration. Symmetry 2018, 10, 132. [CrossRef]

59. Aslam, M.; Arif, O. Testing of grouped product for the Weibull distribution using neutrosophic statistics. Symmetry 2018, 10, 403. [CrossRef]

60. Aslam, M.; Khan, N.; Khan, M. Monitoring the variability in the process using neutrosophic statistical interval method. Symmetry 2018, 10, 562. [CrossRef]

61. Jun, C.-H.; Lee, H.; Lee, S.-H.; Balamurali, S. A variables repetitive group sampling plan under failure-censored reliability tests for Weibull distribution. J. Appl. Stat. 2010, 37, 453-460. [CrossRef] 